Pacific Journal of Mathematics

ON MATRICIALLY NORMED SPACES 


\title{
ON MATRICIALLY NORMED SPACES
}

\author{
EDWARD G. EFFROS AND ZHONG-JIN RUAN
}

\begin{abstract}
Arveson and Wittstock have proved a "non-commutative HahnBanach Theorem" for completely bounded operator-valued maps on spaces of operators. In this paper it is shown that if $T$ is a linear map from the dual of an operator space into a $C^{*}$-algebra, then the usual operator norm of $T$ coincides with the completely bounded norm. This is used to prove that the Arveson-Wittstock theorem does not generalize to "matricially normed spaces". An elementary proof of the Arveson-Wittstock result is presented. Finally a simple bimodule interpretation is given for the "Haagerup" and "matricial" tensor products of matricially normed spaces.
\end{abstract}

1. Introduction. A function space $V$ on a set $X$ is a linear subspace of the bounded complex functions on $X$. With the uniform norm, this is a normed vector space. Conversely, any (complex) normed vector space $V$ may be realized as a function space on the closed unit ball $X$ of the dual space $V^{*}$. Thus one may regard a normed vector space as simply an abstract function space.

An operator space $V$ on a Hilbert space $H$ is a linear subspace of the bounded operators on $H$. For each $n \in \mathbf{N}$, the operator norm associated with $H^{n}$ determines a distinguished norm on the $n \times n$ matrices over $V$. The second author recently gave an abstract characterization for the operator spaces by taking into consideration these systems of matrix norms. The operator spaces $V$ are characterized among the "matricially normed spaces" (see $\S 2$ ), by the " $L^{\infty}$-property": given matrices $v=\left[v_{i j}\right], w=\left[w_{k l}\right]$ with $v_{i j}, w_{k l} \in V$,

$$
\|v \oplus w\|=\max \{\|v\|,\|w\|\} \text {. }
$$

On the other hand, the dual of an operator space is canonically an " $L$ matricially normed space", in the sense that its matrix norms satisfy

$$
\|v \oplus w\|=\|v\|+\|w\| .
$$

In this paper we shall begin a systematic study of the matricially normed spaces. Our main results are:

(a) We show in $\S 2$ that if $\varphi: V \rightarrow W$ is a linear map from an $L^{1}$ matricially normed space to an operator space, then the completely 
bounded norm of $\varphi$ coincides with the usual norm. As a corollary, we conclude that the Arveson-Wittstock Hahn-Banach Theorem for operator spaces cannot be extended to $L^{1}$-matricially normed spaces.

(b) In $\S 3$ we give a direct proof of the Arveson-Wittstock HahnBanach Theorem for operator spaces that uses only the $L^{\infty}$-matricial norm structure, rather than appealing to Wittstock's theory of sublinear set valued functions, or to Paulsen's reduction to the completely positive case (see [1], [14], [9]). Our approach was first considered by Haagerup (unpublished [6]). Using a result of Smith [12], we have been able to substantially simplify his argument.

(c) In $\S 4$ we consider the Haagerup tensor product for matricially normed spaces. In particular, following a suggestion of B. E. Johnson, we consider the corresponding construction in the isomorphic category of normed $\mathscr{F}$-bimodules, $\mathscr{F}$ being the algebra of all finitely non-zero infinite matrices. We show that the Haagerup tensor product corresponds to the projective bimodule tensor product $\hat{\otimes}_{\mathscr{F}}$ (see $(4.1)$ ).

(d) Letting $\mathscr{M}(V, \mathscr{B}(H))$ be the completely bounded maps from a matricially normed space $V$ into $\mathscr{B}(H)$, we have that

$$
\mathscr{M}(V \mathscr{B}(H))=\left[V \otimes_{\mathscr{M}} \mathscr{B}(H)_{*}\right]^{*},
$$

where $\otimes_{\mathscr{M}}$ is the "completely bounded tensor product" (see $\S 3$ and [4]). In $\S 5$ we show that for arbitrary matricially normed spaces, this tensor product is isometric to the projective $\mathscr{F} \otimes \mathscr{F}$-bimodule tensor product $\hat{\otimes}_{\mathscr{G} \otimes \mathscr{F}}$ in Johnson's category (see (5.2)).

We are indebted to Uffe Haagerup for providing us with a copy of [6], and to Barry Johnson for his suggestions regarding $\mathscr{F}$-bimodules.

2. Matricially normed spaces and a Hahn-Banach counter-example. Given normed vector spaces $V$ and $W$, we let $\mathscr{B}(V, W)$ denote the bounded functions from $V$ to $W$ with the usual norm, and $\mathscr{B}(V)=$ $\mathscr{B}(V, V)$. We say that a bounded linear surjection $\varphi: V \rightarrow W$ of normed vector spaces is a quotient map, if it induces an isometry $V / \operatorname{ker} \varphi \rightarrow W$. Equivalently, it maps the open unit ball of $V$ onto that of $W$. If $\varphi: V \rightarrow W$ is an isometric injection, then the adjoint map $\varphi^{*}: W^{*} \rightarrow V^{*}$ is a quotient map.

We let $V \otimes W$ denote the tensor product of arbitrary vector spaces $V$ and $W$. If $V$ and $W$ are normed, we write \|\|$_{\wedge}$ and \|\|$_{\lambda}$ for the projective and injective cross norms on $V \otimes W$ (see [13], §IV.2). These 
are defined by

$$
\begin{aligned}
& \|u\|_{\wedge}=\inf \left\{\sum\left\|v_{i}\right\|\left\|w_{i}\right\|: u=\sum v_{i} \otimes w_{i}\right\}, \\
& \|u\|_{\lambda}=\sup \left\{|p \otimes q(u)|: p \in V^{*}, q \in W^{*},\|p\|=\|q\|=1\right\} .
\end{aligned}
$$

We let $V \hat{\otimes} W$ and $V \otimes_{\lambda} W$ denote the corresponding normed spaces (we do not use the completions in this paper). It is easily verified that if $V \hookrightarrow V_{1}$ is an isometry, the corresponding map $V \otimes_{\lambda} W \hookrightarrow V_{1} \otimes_{\lambda} W$ is again isometric, whereas if $V \rightarrow V_{1}$ is a quotient map, the same is true for the map $V \hat{\otimes} W \rightarrow V_{1} \hat{\otimes} W$.

An operator space $V$ on a Hilbert space $H$ is a linear subspace of $\mathscr{B}(H)$. The latter is a von Neumann algebra, and we denote its predual by $\mathscr{B}(H)_{*}$. Given $\xi, \eta \in H$, we define $\omega_{\xi, \eta} \in \mathscr{B}(H)_{*}$ by

$$
\omega_{\xi, \eta}(b)=b \xi \cdot \eta \text {. }
$$

We let $\mathbf{M}_{m, n}$ (resp., $\mathbf{M}_{n}$ if $m=n$ ) denote the complex $m \times n$ matrices with the usual vector space operations and the operator norm. We let $\varepsilon_{i j} \in \mathbf{M}_{m, n}$ be the matrix units

$$
\varepsilon_{i j}=\left[\begin{array}{c}
j \\
\downarrow \\
\cdots \\
1 \\
0 \cdots 0
\end{array}\right] \leftarrow i
$$

Given a (complex) vector space $V$, we identify the vector space $\mathbf{M}_{m, n}(V)$ of $m \times n$ matrices $\left[v_{i j}\right]\left(v_{i j} \in V\right)$ with the algebraic tensor product $V \otimes \mathbf{M}_{m, n}$, letting $\left[v_{i j}\right] \mapsto \sum v_{i j} \otimes \varepsilon_{i j}$. We regard $V \otimes \mathbf{M}_{n}$ as an $\mathbf{M}_{n}$ bimodule by using the operations

$$
\alpha(v \otimes \mu)=v \otimes \alpha \mu, \quad(v \otimes \mu) \beta=v \otimes \mu \beta .
$$

The corresponding operations in $\mathbf{M}_{n}(V)$ are determined by matrix multiplication. Given $n=n_{1}+\cdots+n_{r}$, we identify $v \in \mathbf{M}_{n}(V)$ with the $r \times r$ matrix of rectangular matrices $\left[\tilde{v}_{p q}\right]$, where

$$
\begin{aligned}
& \tilde{v}_{11}=\left[v_{i j}\right]_{i, j=1, \ldots, n_{1}} \in \mathbf{M}_{n_{1}}(V) \\
& \tilde{v}_{12}=\left[v_{i j}\right]_{i=1, \ldots, n_{1} ; j=n_{1}+1, \ldots, n_{2}} \in \mathbf{M}_{n_{1}, n_{2}}(V)
\end{aligned}
$$

and so on. We also use the notation

$$
v \oplus w=\left[\begin{array}{cc}
v & 0 \\
0 & w
\end{array}\right]
$$


A matricially normed space is a normed vector space $V$ together with an assignment of a norm to each of the matrix spaces $\mathbf{M}_{n}(V)$, such that

$\mathbf{M}_{1} \cdot\left\|v \oplus 0_{r}\right\|=\|v\|\left(v \in \mathbf{M}_{n}(V), r \in \mathbf{N}\right)$.

$\mathbf{M}_{2} .\|\alpha v\| \leq\|\alpha\|\|v\|$ and $\|v \alpha\| \leq\|v\|\|\alpha\|$ for $v \in \mathbf{M}_{n}(V), \alpha \in \mathbf{M}_{n}$. Given $1 \leq p<\infty$, we say that $V$ is an $L^{p}$-matricially normed space if in addition we have that

$\mathrm{L}_{p} \cdot\|v \oplus w\|=\left(\|v\|^{p}+\|w\|^{p}\right)^{1 / p}$ and that it is an $L^{\infty}$-matricially normed space if

$L_{\infty} .\|v \oplus w\|=\max \{\|v\|,\|w\|\}$.

Given a matricially normed space $V$ and a unitary $\alpha \in \mathbf{M}_{n}$, we have from $\mathrm{M}_{2}$ that $\|\alpha v\|=\|v\|=\|v \alpha\|$. In particular, row and column operations on matrices in $\mathbf{M}_{n}(V)$ are isometric. It follows that if $p, q \leq n$, then any of the embeddings of $\mathbf{M}_{p, q}(V)$ in $\mathbf{M}_{n}(V)$ obtained by letting $v \mapsto v^{\prime}$, where $v^{\prime}$ vanishes on $n-p$ and $n-q$ specified rows and columns, determines the same norm on $\mathbf{M}_{p, q}(V)$. In this sense we have norms provided on rectangular matrices over $V$. The relations $M_{1}$ and $M_{2}$ continue to hold for suitable rectangular matrices of elements of $V$ and of $\mathbf{C}$.

In any matricially normed space $V$, we have that if $v=\left[v_{i j}\right] \in$ $\mathbf{M}_{n}(V)$,

$$
\left\|v_{i j}\right\| \leq\|v\| \leq \sum\left\|v_{i j}\right\|
$$

(see [11]). It readily follows that if $V$ is complete, then the same is true for each of the spaces $\mathbf{M}_{n}(V)$. Given a $2 \times 2$ matrix of matrices $v=\left[v_{i j}\right]$, it is also shown in [11] that

$$
\left\|v_{11} \oplus v_{22}\right\| \leq\left\|\left[v_{i j}\right]\right\| .
$$

Given a linear map $\varphi: V \rightarrow W$ of matricially normed spaces, we define a map $\varphi_{m, n}=\varphi \otimes \mathrm{id}: \mathbf{M}_{m, n}(V) \rightarrow \mathbf{M}_{m, n}(W)$ by $\varphi_{m, n}\left(\left[v_{i j}\right]\right)=$ $\left[\varphi\left(v_{i j}\right)\right]$, and we let $\varphi_{n}=\varphi_{n, n}$. We define

$$
\|\varphi\|_{c b}=\sup \left\{\left\|\varphi_{n}\right\|: n \in \mathbf{N}\right\}
$$

and we say that $\varphi$ is completely bounded if $\|\varphi\|_{c b}<\infty$, a complete contraction if $\|\varphi\|_{c b} \leq 1$, and a complete isometry or complete quotient map if each $\varphi_{n}$ is isometric, or a quotient map, respectively. It is immediate that if $p \geq \max \{m, n\},\left\|\varphi_{m, n}\right\| \leq\left\|\varphi_{p}\right\|$. We let $\mathscr{M}(V, W)$ denote the normed vector space of all completely bounded linear maps $\varphi: V \rightarrow W$ together with the corresponding norm \|\|$_{c b}$. It should be noted that we do not attempt to place a matricial norm structure on $\mathscr{M}(V, W)$ in this paper. 
As we remarked in $\S 1$, any operator space $V$ is provided with matrix norms, namely, we may let $\mathbf{M}_{n}(V)$ have the relative norm in $\mathbf{M}_{n} \mathscr{B}(H) \cong \mathscr{B}\left(H^{n}\right)$. It is trivial that with these norms $V$ is an $L^{\infty}$. matricially normed space.

If $W$ is a vector subspace of a matricially normed space $V$, then identifying $\mathbf{M}_{n}(W)$ with a subspace of $\mathbf{M}_{n}(V)$, the relative norms determine a matricial norm structure on $W$, and we say that $W$ is a matricially normed subspace of $V$.

If $V$ is matricially normed, then regarding $\mathbf{M}_{n}\left(V^{*}\right)$ as the dual of $\mathbf{M}_{n}(V)$ under the pairing

$$
\left\langle\left[v_{i j}\right],\left[f_{i j}\right]\right\rangle=\sum f_{i j}\left(v_{i j}\right),
$$

$V^{*}$ is matricially normed by the dual norms on $\mathbf{M}_{n}\left(V^{*}\right)$ (see [11]). We call $V^{*}$ the dual matricially normed space of $V$. If $V \subseteq \mathscr{B}(H)$ is an operator space, and we are given $\xi, \eta \in H^{n}$, then for any $v \in \mathbf{M}_{n}(V)$,

$$
\omega_{\xi, \eta}(v)=v \xi \cdot \eta=\sum v_{i j} \xi_{j} \cdot \eta_{i}=\sum \omega_{\xi_{j}, \eta_{i}}\left(v_{i j}\right),
$$

hence with the above convention,

$$
\omega_{\xi, \eta}=\left[\omega_{\xi, \eta}\right] .
$$

It will be convenient to regard the dual of $\mathbf{M}_{n}$ as also being the predual, and we denote it by $\mathbf{M}_{n^{*}}$.

Finally if $W$ is a closed subspace of a matricially normed space $V$, then it follows from (2.1) that $\mathbf{M}_{n}(W)$ is closed in $\mathbf{M}_{n}(V)$. Identifying $\mathbf{M}_{n}(V / W)$ with $\mathbf{M}_{n}(V) / \mathbf{M}_{n}(W)$, we may let $\mathbf{M}_{n}(V / W)$ have the quotient norm for each $n$. It is readily verified that $V / W$ is thereby matricially normed. This is called the quotient matricially normed space. If $V$ is an $L^{p}$-matricially normed space then subspaces and quotients again have that property (for the latter, see (2.2)). In general we have that a map $\varphi: V \rightarrow W$ is a complete quotient map if and only if it induces a complete isometry of $V / \operatorname{ker} \varphi$ onto $W$.

THEOREM 2.1. Given a normed vector space $V$, the norms on the matrix spaces $V \otimes_{\lambda} \mathbf{M}_{n}$ determine an $L^{\infty}$-matricial structure on $V$, whereas the norms on the matrix spaces $V \hat{\otimes} \mathbf{M}_{n^{*}}$ determine an $L^{1}$ matricial structure on $V$.

Proof. To prove the first assertion, we embed $V$ into $l^{\infty}(X)$ for some set $X$, and then find a faithful representation of $l^{\infty}(X)$ 
on a Hilbert space $H$. This provides $l^{\infty}(X)$ with an $L^{\infty}$-matricial structure, and we let $V$ have the relative structure. The matrix norms for $l^{\infty}(X)$ are determined by the isometries:

$$
\mathbf{M}_{n}\left(l^{\infty}(X)\right) \cong l^{\infty}(X) \otimes_{\lambda} \mathbf{M}_{n}
$$

(see [13], Th. IV. 4.14). Since the maps $V \otimes_{\lambda} \mathbf{M}_{n} \hookrightarrow l^{\infty}(X) \otimes_{\lambda} \mathbf{M}_{n}$ are isometric, the relative $L^{\infty}$-matricial structure is given by the isometries:

$$
\mathbf{M}_{n}(V) \cong V \otimes_{\lambda} \mathbf{M}_{n} .
$$

For the second, we let $X$ be the open unit ball of $V$, and we define $l(X) \subseteq l^{1}(X)$ to be the functions on $X$ vanishing off finitely many points, together with the $l^{1}$ norm. Letting $\delta(x)$ denote the characteristic function of the singleton $\{x\}$, the map

$$
\theta: l(X) \rightarrow V: \sum \alpha_{k} \delta\left(x_{k}\right) \mapsto \sum \alpha_{k} x_{k}
$$

is a quotient map of normed vector spaces. Representing $l^{\infty}(X)$ as a von Neumann algebra on a Hilbert space $H$, it follows that $l^{1}(X)$ is then isometric to the predual of this von Neumann algebra, and thus $l(X)$ inherits a corresponding $L^{1}$-matricial norm structure. We let $V \cong l(X) / \operatorname{ker} \theta$ have the quotient $L^{1}$-matricial structure. The matrix norms on $l(X)$ are determined by the isometries

$$
\mathbf{M}_{n}(l(X)) \cong l(X) \hat{\otimes} \mathbf{M}_{n^{*}}
$$

(see [13] Th. IV.7.17, and p. 261). Since

$$
l(X) \hat{\otimes} \mathbf{M}_{n^{*}} \rightarrow V \hat{\otimes} \mathbf{M}_{n^{*}}
$$

is a quotient map, the matricial norm on $V$ are given by the isometries

$$
\mathbf{M}_{n}(V) \cong V \hat{\otimes} \mathbf{M}_{n^{*}}
$$

The following results were proved in [11]:

THEOREM 2.2. Suppose that $V$ is matricially normed. Then

(1) $V$ is completely isometric to an operator space if and only if it is an $L^{\infty}$-matricially normed space.

(2) $V$ is an $L^{1}$-matricially normed space if and only if $V^{*}$ is an $L^{\infty}$-matricially normed space. 
In light of (1), we shall also refer to $L^{\infty}$-matricially normed spaces as abstract operator spaces, or more simply, operator spaces.

COROLlary 2.3. Suppose that $V$ is a dual $L^{1}$-matricially normed space. Then it is a quotient of $\mathscr{B}(H)_{*}$ for some Hilbert space $H$.

Proof. We may assume that $V=\left(V_{*}\right)^{*}$ for some matricially normed space $V_{*}$. Since the canonical map $j: V_{*} \rightarrow V^{*}$ is completely isometric, the dual $j^{*}: V^{* *} \rightarrow V$ is a complete quotient map. From Theorem 2.2, $V^{*}$ is an $L^{\infty}$-matricially normed space, and there is a complete isometry $\varphi: V^{*} \rightarrow \mathscr{B}(K)$ for some Hilbert space $K$. Thus $\varphi^{*}: \mathscr{B}(K)^{*} \rightarrow V^{* *}$ is a quotient map. We have that $\mathscr{B}(K)^{*}=\mathscr{R}_{*}$, where $\mathscr{R}=\mathscr{B}(K)^{* *}$ is a von Neumann algebra on some Hilbert space $H$. Since $\mathscr{R}$ is weak ${ }^{*}$ closed in $\mathscr{B}(H), \mathscr{R}_{*} \cong \mathscr{B}(H)_{*} / \mathscr{R}_{\perp}$, where $\mathscr{R}_{\perp}$ is the annihilator of $\mathscr{R}$. The composition of these quotient maps gives us a quotient map $\mathscr{B}(H)_{*} \rightarrow V$.

It was shown in [11] that if $A$ and $B$ are $C^{*}$-algebras, then there are no completely bounded maps from $A$ into $B^{*}$. This is even the case if $A=B=$ C. In the reverse direction we have:

THEOREM 2.4. Suppose $V$ is an $L^{1}$-matricially normed space and $W$ is an operator space. Then for any linear map $\varphi: V \rightarrow W$ we have that

$$
\|\varphi\|_{c b}=\|\varphi\| .
$$

Proof. It suffices to show that if $\|\varphi\|<\infty$, then for each $n \in \mathbf{N}$, $\left\|\varphi_{n}\right\| \leq\|\varphi\|$. Let us first assume that $V=\mathscr{B}(H)_{*}$ for some Hilbert space $H$, and that $W \subseteq \mathscr{B}(K)$. Each function $f \in \mathscr{B}(H)_{*}$ with $\|f\| \leq 1$ is a norm limit of convex combinations of functions of the form $\omega_{\xi, \eta}$ (see [3] $\S I .3 .3$, proof of Lemma 3). We may apply this to $\mathbf{M}_{n}\left(\mathscr{B}(H)_{*}\right)=\left[\mathbf{M}_{n}(\mathscr{B}(H))\right]_{*}$. Given vectors $\theta, \zeta \in K^{n}$, the function $f \mapsto\left|\varphi_{n}(f) \theta \cdot \zeta\right|$ is convex on the unit ball of $\mathbf{M}_{n}\left(\mathscr{B}(H)_{*}\right)$, and thus assumes its maximum on functions of the form $\omega_{\xi, \eta}, \xi, \eta \in H^{n}$ :

$$
\begin{aligned}
\left\|\varphi_{n}\right\|=\sup \left\{\left|\varphi_{n}\left(\omega_{\xi, \eta}\right) \theta \cdot \zeta\right|:\|\xi\|,\|\eta\|,\|\theta\|,\|\zeta\|\right. & \leq 1, \\
& \left.\xi, \eta \in H^{n}, \theta, \zeta \in K^{n}\right\} .
\end{aligned}
$$


Using (2.3), we have

$$
\begin{aligned}
& \left|\varphi_{n}\left(\omega_{\xi, \eta}\right) \theta \cdot \zeta\right|=\left|\left[\varphi\left(\omega_{\xi_{j}, \eta_{t}}\right)\right]\left(\theta_{j}\right) \cdot\left(\zeta_{i}\right)\right| \\
& \quad=\left|\sum_{i, j} \varphi\left(\omega_{\xi_{j}, \eta_{i}}\right) \theta_{j}, \zeta_{i}\right| \\
& \quad \leq q\|\varphi\| \sum_{i, j}\left\|\xi_{j}\right\|\left\|\eta_{i}\right\|\left\|\theta_{j}\right\|\left\|\zeta_{i}\right\| \\
& \quad=\|\varphi\|\left[\sum_{\left.\left\|\xi_{j}\right\|\left\|\theta_{j}\right\|\right]\left[\sum\left\|\eta_{i}\right\|\left\|\zeta_{i}\right\|\right]}\right. \\
& \quad \leq\|\varphi\|\left[\sum\left\|\xi_{i}\right\|^{2}\right]^{1 / 2}\left[\sum\left\|\theta_{i}\right\|^{2}\right]^{1 / 2}\left[\sum\left\|\eta_{i}\right\|^{2}\right]^{1 / 2}\left[\sum\left\|\zeta_{i}\right\|^{2}\right]^{1 / 2} \\
& \quad=\|\varphi\|\|\xi\|\|\theta\|\|\eta\|\|\zeta\|,
\end{aligned}
$$

and thus $\left\|\varphi_{n}\right\| \leq\|\varphi\|$.

Now let us suppose that $V$ is a dual $L^{1}$-matricially normed space. From Corollary 2.3 there is a quotient map $\pi: \mathscr{B}(H)_{*} \rightarrow V$ in the category of matricially normed spaces, i.e., each of the maps $\pi_{n}$ : $\mathbf{M}_{n}\left(\mathscr{B}(H)_{*}\right) \rightarrow \mathbf{M}_{n}(V)$ is a quotient map. Since $\pi_{n}$ maps the open unit ball onto the open unit ball of the image, it follows that

$$
\left\|\varphi_{n}\right\|=\left\|\varphi_{n} \circ \pi_{n}\right\|=\left\|(\varphi \circ \pi)_{n}\right\|=\|\varphi \circ \pi\|=\|\varphi\| .
$$

Finally suppose that $V$ is a general $L^{1}$-matricially normed space. Since $V^{* *}$ is a dual $L^{1}$-matricially normed space, the map $\varphi^{* *}: V^{* *} \rightarrow$ $W^{* *}$ satisfies $\left\|\varphi^{* *}\right\|_{c b}=\left\|\varphi^{* *}\right\|$. For any $n$ we have that $\left(\varphi^{* *}\right)_{n}=\left(\varphi_{n}\right)^{* *}$, hence $\left\|\left(\varphi^{* *}\right)_{n}\right\|=\left\|\varphi_{n}\right\|$, and $\|\varphi\|_{c b}=\left\|\varphi^{* *}\right\|_{c b}=\left\|\varphi^{* *}\right\|=\|\varphi\|$.

COROLLARY 2.5. Given $L^{1}$-matricially normed spaces $V \subseteq W, a$ completely bounded map $\varphi: V \rightarrow \mathbf{M}_{2}$ need not have an extension $\psi: W \rightarrow \mathbf{M}_{2}$ satisfying $\|\psi\|_{c b}=\|\varphi\|_{c b}$.

Proof. $\mathbf{M}_{2}$ is not injective as a normed vector space since it is not isometric to $l_{4}^{\infty}$ (this is a consequence of [7], Th. 7), which is the only 4dimensional injective normed vector space (see [8] $\S 3.11$, Th. 6). Thus we may find normed vector spaces $V \subseteq W$ and a map $\varphi: V \rightarrow \mathbf{M}_{2}$ which does not have an extension $\psi: W \rightarrow \mathbf{M}_{2}$ with $\|\psi\|=\|\varphi\|$. From above we may extend the norm structure on $W$ to an $L^{1}$-matricial norm structure. Letting $V$ have the relative matricial norm structure, $V$ and $W$ are $L^{1}$-matricial norm spaces. Since $\|\varphi\|_{c b}=\|\varphi\|$ and $\|\psi\|_{c b}=\|\psi\|$, we cannot find an extension $\psi: W \rightarrow \mathbf{M}_{2}$ with $\|\psi\|_{c b}=\|\varphi\|_{c b}$. 
It should be noted that in the Proof of Corollary 2.5, although $M_{n}(W) \cong W \widehat{\otimes} M_{n *}$ we cannot conclude that $M_{n}(V) \cong V \widehat{\otimes} M_{n *}$.

3. The Hahn-Banach Theorem for operator spaces. Consider the action of $\mathbf{M}_{p}\left(\mathbf{M}_{n}\right)$ on $\left(\mathbf{C}^{n}\right)^{p}$. Under the identification of $\mathbf{M}_{n} \otimes \mathbf{M}_{p} \cong$ $\mathbf{M}_{p}\left(\mathbf{M}_{n}\right)$ we have that

$$
\begin{gathered}
\alpha \otimes I_{p}=\sum_{i} \alpha \otimes \varepsilon_{i i}=\left[\begin{array}{l}
\alpha \cdots 0 \\
0 \cdots \alpha
\end{array}\right], \\
I_{n} \otimes \beta=\sum_{i j} \beta_{i j} I_{n} \otimes \varepsilon_{i j}=\left[\begin{array}{c}
\beta_{11} I_{n} \cdots \beta_{1 p} I_{n} \\
\beta_{p 1} I_{n} \cdots \beta_{p p} I_{n}
\end{array}\right] .
\end{gathered}
$$

Thus given $\xi \in\left(\mathbf{C}^{n}\right)^{p}$,

$$
\left(\alpha \otimes I_{p}(\xi)\right)_{i}=\alpha \xi_{i}, \quad\left(I_{n} \otimes \beta(\xi)\right)_{i}=\sum_{j} \beta_{i j} \xi_{j}
$$

The following result is due to $R$. Smith [12]. We have included a proof that may be more familiar to operator algebraists, and that has an obvious extension to finite von Neumann algebras.

LEMMA 3.1. Suppose that $p \geq n$, and the $\xi_{0}$ is a vector in $\left(\mathbf{C}^{n}\right)^{p}$. Then there exists a unitary matrix $U \in \mathbf{M}_{p}$ and a vector $\xi \in\left(\mathbf{C}^{n}\right)^{n}$ such that $\left(I_{n} \otimes U\right) \xi_{0}=\xi \oplus o_{p-n}$.

Proof. The representation of $\alpha \mapsto \alpha \otimes I_{n}$ of $\mathbf{M}_{n}$ on $\left(\mathbf{C}^{n}\right)^{n}$ has a separating vector, and thus any state on $\mathbf{M}_{n}$ is a vector state in this representation, i.e., it has the form $\omega_{\xi}$ for some vector $\xi \in\left(\mathbf{C}^{n}\right)^{n}$. it follows that given an arbitrary vector $\xi_{0} \in\left(\mathbf{C}^{n}\right)^{p}=\left(\mathbf{C}^{n}\right)^{n} \oplus\left(\mathbf{C}^{n}\right)^{p-n}$, we choose $\xi \in\left(\mathbf{C}^{n}\right)^{n}$ with $\omega_{\xi_{0}, \xi_{0}}=\omega_{\xi \oplus 0, \xi \oplus 0}$ on $\mathbf{M}_{n}$. From elementary operator algebra theory, the map $\left(\alpha \otimes I_{p}\right)\left(\xi_{0}\right) \mapsto\left(\alpha \otimes I_{p}\right)(\xi \oplus 0)$ extends to a partial isometry $U_{0}^{\prime}$ of $\mathbf{M}_{n} \otimes I_{p}\left(\xi_{0}\right)$ onto $\mathbf{M}_{n} \otimes I_{p}(\xi \oplus 0)$, which lies in $\left(\mathbf{M}_{n} \otimes I_{p}\right)^{\prime}$. Letting $E^{\prime}$ and $F^{\prime}$ be the domain and range projections of $U_{0}^{\prime}, E^{\prime}$ and $F^{\prime}$ are equivalent in $\left(\mathbf{M}_{n} \otimes I_{p}\right)^{\prime}$, and thus the same is true for $I-E^{\prime}$ and $I-F^{\prime}$. Adding a corresponding partial isometry to $U_{0}^{\prime}$, we obtain a unitary $U_{0}$ in $\left(\mathbf{M}_{n} \otimes I_{p}\right)^{\prime}$ with $U_{0} \xi_{0}=\xi \oplus 0$. Since $\left(\mathbf{M}_{n} \otimes I_{p}\right)^{\prime}=I_{n} \otimes \mathbf{M}_{p}, U_{0}$ must have the form $I_{n} \otimes U$, with $U$ a unitary in $\mathbf{M}_{p}$.

Generalizing [12], we have:

LEMMA 3.2. Suppose that $V$ is a matricially normed space. Then given a linear map $\varphi: V \rightarrow \mathbf{M}_{n}$, we have that $\|\varphi\|_{c b}=\left\|\varphi_{n}\right\|$. 
Proof. Given $p>n$ and unit vectors $\xi, \eta \in\left(\mathbf{C}^{n}\right)^{p}$, we may choose unitary matrices $U, V \in \mathbf{M}_{p}$ and unit vectors $\xi^{\prime}, \eta^{\prime} \in\left(\mathbf{C}^{n}\right)^{n}$ such that $\xi=\left(I_{n} \otimes U\right)\left(\xi^{\prime} \oplus o_{p-n}\right)$ and $\eta=\left(I_{n} \otimes V\right)\left(\eta^{\prime} \oplus o_{p-n}\right)$. Letting $E=$ $\left[\begin{array}{ll}I_{n} & O\end{array}\right]$ and $U^{\prime}=I_{n} \otimes U E^{*}, V^{\prime}=I_{n} \otimes V E^{*}$ (these are $p \times n$ matrices over $\left.\mathbf{M}_{n}\right)$, it follows that for $v \in \mathbf{M}_{p}(V)$ with $\|v\| \leq 1$,

$$
\begin{aligned}
\left|\varphi \otimes I_{p}(v) \xi \cdot \eta\right| & =\left|\varphi \otimes I_{n}\left(V^{* *} v U^{\prime}\right) \xi^{\prime} \cdot \eta^{\prime}\right| \\
& \leq\left\|\varphi_{n}\right\|\left\|V^{\prime *} v U^{\prime}\right\|\left\|\xi^{\prime}\right\|\left\|\eta^{\prime}\right\| \leq\left\|\varphi_{n}\right\| .
\end{aligned}
$$

COROLlaRy 3.3. If $V$ is matricially normed and $f \in V^{*}$, then $\|f\|_{c b}$ $=\|f\|$.

Given a matricially normed space $V$ an operator space $W$ and elements $f \in V^{*}$ and $w \in W$, we define a linear map $\theta_{f \otimes w}: V \rightarrow W$ by $\theta_{f \otimes w}(v)=f(v) w$.

COROLlARY 3.4. Given a matricially normed space $V$, and operator space $W$ and elements $f \in V^{*}, w \in W$ we have that $\left\|\theta_{f \otimes w}\right\|_{c b} \leq$ $\|f\|\|w\|$.

Proof. We have that if $v \in \mathbf{M}_{n}(V),\|v\| \leq 1$,

$$
\begin{aligned}
\left\|\left(\theta_{f \otimes w}\right)_{n}(v)\right\| & =\left\|\left[f\left(v_{i j}\right) w\right]\right\|=\left\|f_{n}(v)(w \oplus \cdots \oplus w)\right\| \\
& \leq\left\|f_{n}(v)\right\|\|w \oplus \cdots \oplus w\| \leq\|f\|\|w\| .
\end{aligned}
$$

Let us suppose that $V$ is a matricially normed space. We have a natural pairing between $\mathscr{M}\left(V, \mathbf{M}_{n}\right)$ and the algebraic tensor product $V \otimes \mathbf{M}_{n^{*}}$ defined by $\langle\varphi, v \otimes g\rangle=g(\varphi(g))$. In fact $\mathscr{M}\left(V, \mathbf{M}_{n}\right)$ is in this manner isometric to the dual of $V \otimes \mathbf{M}_{n^{*}}$ when the latter is provided with a suitable norm \|\|$_{\mathscr{M}}$ described below.

Given $v \in \mathbf{M}_{n}(V)$ and $g \in \mathbf{M}_{n}\left(\mathbf{M}_{n^{*}}\right)$, we define $v \times g \in V \otimes \mathbf{M}_{n^{*}}$ by

$$
v \times g=\sum v_{i j} \otimes g_{i j} .
$$

A simple calculation shows that for any $\alpha \in \mathbf{M}_{m, n}, \beta \in \mathbf{M}_{n, m}, v \in$ $\mathbf{M}_{n}(V)$ and $g \in \mathbf{M}_{m}\left(\mathbf{M}_{n^{*}}\right)$, we have

$$
(\alpha v \beta) \times g=v \times\left(\alpha^{\text {tr }} g \beta^{\text {tr }}\right),
$$

where tr indicates the transposed matrix. For any $\varphi \in \mathscr{M}\left(V, \mathbf{M}_{n}\right)$,

$$
\left\langle\varphi_{n}(v), g\right\rangle=\sum\left\langle\varphi\left(v_{i j}\right), g_{i j}\right\rangle=\langle\varphi, v \times g\rangle,
$$


and thus

$$
\begin{aligned}
& \|\varphi\|_{c b}=\left\|\varphi_{n}\right\| \\
& \quad=\sup \left\{\left|\left\langle\varphi_{n}(v), g\right\rangle\right|:\|v\|,\|g\| \leq 1, v \in \mathbf{M}_{n}(V), g \in \mathbf{M}_{n}\left(\mathbf{M}_{n^{*}}\right)\right\} \\
& \quad=\sup \left\{|\langle\varphi, v \times g\rangle|:\|v\|,\|g\| \leq 1, v \in \mathbf{M}_{n}(V), g \in \mathbf{M}_{n}\left(\mathbf{M}_{n^{*}}\right)\right\} .
\end{aligned}
$$

We define the completely bounded norm \|\|$_{\mathscr{M}}$ on $V \otimes \mathbf{M}_{n^{*}}$ by

$$
\begin{aligned}
& \|F\|_{\mathscr{M}}=\inf \left\{\sum\left\|v_{i}\right\|\left\|g_{i}\right\|: F=\sum v_{i} \times g_{i}, v_{i} \in \mathbf{M}_{n}(V),\right. \\
& \left.\qquad g_{i} \in \mathbf{M}_{n}\left(\mathbf{M}_{n^{*}}\right)\right\} .
\end{aligned}
$$

It is evident that this is a seminorm. To see that \|\|$_{\mathscr{M}}$ is a norm, let us suppose that $F=\sum w_{i} \otimes h_{i} \neq 0, w_{i} \in V, h_{i} \in \mathbf{M}_{n^{*}}$. We may assume that the $h_{i}$ form a basis for $\mathbf{M}_{n^{*}}$, and that $w_{1} \otimes h_{1} \neq 0$. Letting $\beta_{i} \in \mathbf{M}_{n}$ be a dual basis for the $h_{i}$, and choosing $f \in V^{*}$ with $f\left(w_{1}\right) \neq 0$, we have that $\left\langle\theta_{f \otimes \beta_{1}}, F\right\rangle=f\left(w_{1}\right) \neq 0$. Since we have

$$
\left|\left\langle\theta_{f \otimes \beta_{1}}, F\right\rangle\right| \leq\left\|\theta_{f \otimes \beta_{1}}\right\|_{c b}\|F\|_{\mathscr{M}},
$$

it follows that $\|F\|_{\mathscr{M}} \neq 0$.

We let $V \otimes_{\mathscr{M}} \mathbf{M}_{n^{*}}$ denote $V \otimes \mathbf{M}_{n^{*}}$ with the completely bounded norm, and we call it the completely bounded tensor product. It follows from (3.2) that if $\varphi \in \mathscr{M}\left(V, \mathbf{M}_{n}\right)$ and $F \in V \otimes_{\mathscr{M}} \mathbf{M}_{n^{*}}$ we have

$$
\|\varphi\|_{c b}=\sup \left\{|\langle\varphi, F\rangle|:\|F\|_{\mathscr{M}} \leq 1, F \in V \otimes_{\mathscr{M}} \mathbf{M}_{n^{*}}\right\}
$$

and thus

$$
\mathscr{M}\left(V, \mathbf{M}_{n}\right) \cong\left[V \otimes \mathbf{M}_{n^{*}}\right]^{*}
$$

We say that a function $g=\left[g_{i j}\right] \in \mathbf{M}_{n}\left(\mathbf{M}_{n^{*}}\right)$ is non-singular if the $g_{i j}$ are linearly independent functions in $\mathbf{M}_{n^{*}}$.

LemMA 3.5. Given $g \in \mathbf{M}_{n}\left(\mathbf{M}_{n^{*}}\right)$ with $g=\omega_{\xi, \eta}$ where $\xi, \eta \in\left(\mathbf{C}^{n}\right)^{n}$, $g$ is non-singular if and only if $\xi$ and $\eta$ are cyclic vectors for $\mathbf{M}_{n} \otimes I_{n}$.

Proof. $g$ will be non-singular if and only if the entries $g_{i j}=\omega_{\xi_{,} \eta_{1}}$ $\operatorname{span} \mathbf{M}_{n^{*}}$, or equivalently, $g_{i j}(\alpha)=0$ for all $i, j$ implies that $\alpha=0$. Thus $g$ is non-singular if and only if

$$
\alpha \xi_{j} \cdot \eta_{i}=0 \quad \text { for all } i, j \Rightarrow \alpha=0 .
$$

It is clear that a necessary and sufficient condition for this is that the $\xi_{j}$ and the $\eta_{i}$ span $\mathbf{C}^{n}$. The $\xi_{j}$ are linearly independent if and only if given any $n$ vectors $\zeta_{j}$, there is a matrix $\beta \in \mathbf{M}_{n}$ with $\beta \xi_{j}=\zeta_{j}$, or letting 
$\zeta=\left(\zeta_{1}, \ldots, \zeta_{n}\right)$ be the corresponding vector in $\left(\mathbf{C}^{n}\right)^{n}, \zeta=\left(\beta \otimes I_{n}\right) \xi$. Thus the $\xi_{j}$ span $\mathbf{C}^{n}$ if and only if $\xi$ is cyclic for $\mathbf{M}_{n} \otimes I_{n}$. Since the same applies to $\eta$, we are done.

From the definition of the norm, if $F \in V \otimes_{\mathscr{M}} \mathbf{M}_{n^{*}}$ satisfies $\|F\|<1$, then there is a finite sum representation

$$
F=\sum t_{i} v_{i} \times g_{i}, \quad\left(v_{i} \in \mathbf{M}_{n}(V), g_{i} \in \mathbf{M}_{n}\left(\mathbf{M}_{n^{*}}\right)\right)
$$

where $\left\|v_{i}\right\|<1,\left\|g_{i}\right\|=1, t_{i} \geq 0$, and $\sum t_{i}=1$. The following may be regarded as the major distinguishing feature of the operator spaces. It was first proved by Haagerup [6] using a rather different argument.

LEMMA 3.6. Suppose that $V$ is an operator space and that $F \in V \otimes$ $\mathbf{M}_{n^{*}}$ satisfies $\|F\|<1$. Then $F=v \times g$ where $v \in \mathbf{M}_{n}(V)$ and $g \in$ $\mathbf{M}_{n}\left(\mathbf{M}_{n^{*}}\right)$ satisfy $\|v\|<1,\|g\|=1$. Furthermore, $g$ may be chosen non-singular.

Proof. We may assume that $F$ has the representation (3.4). Since any function $g_{i} \in \mathbf{M}_{n}\left(\mathbf{M}_{n^{*}}\right)$ with $\left\|g_{i}\right\|=1$ is a convex combination of functionals of the form $\omega_{\xi_{,}, \eta_{j}}$, where $\xi_{j}, \eta_{j}$ are unit vectors in $\left(\mathbf{C}^{n}\right)^{n}$, $F$ has the representation

$$
F=\sum_{k=1}^{p} t_{k}\left(v_{k} \times \omega_{\xi_{k}, \eta_{k}}\right),
$$

with $\xi_{k}, \eta_{k}$ unit vectors in $\left(\mathbf{C}^{n}\right)^{n}, v_{k} \in \mathbf{M}_{n}(V)$ satisfying $\left\|v_{k}\right\|<1$, $t_{k} \geq 0$, and $\sum t_{k}=1$. Since $V$ is an operator space, $\tilde{v}=v_{1} \oplus \cdots \oplus v_{p} \in$ $\mathbf{M}_{n p}(V)$ satisfies $\|\tilde{v}\|<1$. We have that

$$
F=\tilde{v} \times \omega_{\xi_{0} \eta_{0}}
$$

where

$$
\xi_{0}=\left(t_{1}^{1 / 2} \xi_{1}\right) \oplus \cdots \oplus\left(t_{p}^{1 / 2} \xi_{p}\right), \quad \eta_{0}=\left(t_{1}^{1 / 2} \eta_{1}\right) \oplus \cdots \oplus\left(t_{p}^{1 / 2} \eta_{p}\right)
$$

are unit vectors in $\left(\mathbf{C}^{n}\right)^{n p}$. From Lemma 3.1, we may choose unitary matrices $U, V \in \mathbf{M}_{n p}$ and unit vectors $\xi, \eta \in\left(\mathbf{C}^{n}\right)^{n}$ such that

$$
\begin{array}{ll}
\xi_{0}=\left(I_{n} \otimes U\right) \xi^{\prime}, & \xi^{\prime}=\xi \oplus 0 \in\left(\mathbf{C}^{n}\right)^{n} \oplus\left(\mathbf{C}^{n}\right)^{n p-n}, \\
\eta_{0}=\left(I_{n} \otimes V\right) \eta^{\prime}, & \eta^{\prime}=\eta \oplus 0 \in\left(\mathbf{C}^{n}\right)^{n} \oplus\left(\mathbf{C}^{n}\right)^{n p-n} .
\end{array}
$$

It follows that

$$
\omega_{\xi_{0} \eta_{0}}=\bar{V} \omega_{\xi^{\prime} \eta^{\prime}} U^{\mathrm{tr}}
$$


since $\left(\xi_{0}\right)_{j}=\sum_{l=1}^{n p} U_{j l} \xi_{l}^{\prime}$, and $\left(\eta_{0}\right)_{i}=\sum_{k=1}^{n p} V_{i k} \eta_{k}^{\prime}$ imply that

$$
\left(\omega_{\xi_{0} \eta_{0}}\right)_{i j}=\sum_{k, l=1}^{n p} U_{j l} \bar{V}_{i k} \omega_{\xi_{l}^{\prime}, \eta_{k}^{\prime}} .
$$

But as elements of $\mathbf{M}_{n^{*}}$, we have that $\omega_{\xi^{\prime}, \eta_{i}^{\prime}}=\omega_{\xi_{\mu}, \eta_{i}}$ for $i, j \leq n$, and otherwise they are zero. Thus

$$
\omega_{\xi^{\prime}, \eta^{\prime}}=E^{*} \omega_{\xi, \eta} E
$$

where $E=\left[I_{n} O_{p-n}\right]$, and we have from (3.1),

$$
F=\tilde{v} \times \bar{V} E^{*} \omega_{\xi, \eta} E U^{\mathrm{tr}}=E V^{*} \tilde{v} U E^{*} \times \omega_{\xi, \eta}=v \times \omega_{\xi, \eta},
$$

where $v=E V^{*} \tilde{v} U E^{*} \in \mathbf{M}_{n}(V)$ satisfies $\|v\| \leq\|\tilde{v}\|<1$.

Our next task is to perturb $\xi, \eta \in\left(\mathbf{C}^{n}\right)^{n}$ so that the resulting linear function $\omega_{\xi, \eta}=\left[\omega_{\xi_{s}, \eta_{l}}\right]$ is non-singular, i.e., so that both $\xi$ and $\eta$ are cyclic for $\mathbf{M}_{n} \otimes I$. Letting $e_{i}$ be the canonical basis for $\mathbf{C}^{n}$, we have that $e=\left(e_{1}, \ldots, e_{n}\right)$ is cyclic for $\mathbf{M}_{n} \otimes I$. Given $t$ with $0 \leq t<1$, we let $v_{t}=(1-t)^{-2} v$ and

$$
\xi_{t}=(1-t) \xi \oplus t e, \quad \eta_{t}=(1-t) \eta \oplus t e,
$$

which are vectors in $\left(\mathbf{C}^{n}\right)^{2 n}$. We then have that

$$
F=(1-t)^{2}\left(v_{t} \times \omega_{\xi, \eta}\right)+t^{2}\left(0 \times \omega_{e, e}\right)=\left(v_{t} \oplus 0\right) \times \omega_{\xi_{t}, \eta_{t}} .
$$

Fixing $t$, we have from Lemma 3.1 that there exist unitaries $U, V \in$ $\mathbf{M}_{2 n}$ such that $(I \otimes U) \xi_{t}=\xi^{\prime} \oplus 0$ and $(I \otimes V) \eta_{t}=\eta^{\prime} \oplus 0$, and thus as above

$$
F=w \times \omega_{\xi^{\prime}, \eta^{\prime}}
$$

where $\|w\| \leq\left\|v_{t}\right\|$. For small $t$, we will have that $\left\|\xi_{t}\right\|,\left\|\eta_{t}\right\|$ are close to 1 and $\left\|v_{t}\right\|$ is close to $\|v\|$. It thus suffices to show that we can choose arbitrarily small $t>0$ with $\xi^{\prime}, \eta^{\prime}$ cyclic for $\mathbf{M}_{n} \otimes I_{n}$, since even after we normalize $\xi^{\prime}$ and $\eta^{\prime}$ to be unit vectors, absorbing the constants into $w$, we will still have that $\|w\|<1$. $\xi^{\prime}$ will be cyclic for $\mathbf{M}_{n} \otimes I_{n}$ if and only if the $n^{2}$ vectors $\varepsilon_{i j} \otimes I_{2 n}\left(\xi^{\prime} \oplus 0\right)$ span an $n^{2}$ dimensional space, or since $I_{n} \otimes U$ commutes with the action of $\mathbf{M}_{n} \otimes I_{2 n}$, if and only if the vectors $\varepsilon_{i j} \otimes I_{2 n} \xi_{t}$ are linearly independent in $\left(\mathbf{C}^{n}\right)^{2 n}$. Using exterior products, this is equivalent to

$$
R(t)=\left(\varepsilon_{11} \otimes I_{2 n} \xi_{t}\right) \wedge \cdots \wedge\left(\varepsilon_{n n} \otimes I_{2 n} \xi_{t}\right) \neq 0 .
$$

Given a basis $d_{k}$ for $\left(\mathbf{C}^{n}\right)^{2 n}$, the exterior products

$$
d_{K}=d_{k_{1}} \wedge \cdots \wedge d_{k_{n^{2}}}
$$


for $n^{2}$-tuples $K=\left(k_{1}, \ldots k_{n^{2}}\right)$ with $k_{1}<\cdots<k_{n^{2}}$, form a basis for $\bigwedge^{n^{2}}\left(\mathbf{C}^{n}\right)^{2 n}$. Thus we have unique coefficients $P_{K}(t)$ with

$$
R(t)=\sum_{K} P_{K}(t) d_{K}
$$

Letting $t$ vary in $\mathbf{R}$, the $P_{K}(t)$ are polynomials in $t$. Since the vectors $\left(\varepsilon_{i j} \otimes I_{n}\right) e\left(1 \leq i, j \leq n^{2}\right)$ are linearly independent, the same is true for the vectors $0 \oplus\left(\varepsilon_{i j} \otimes I_{n}\right) e\left(1 \leq i, j \leq n^{2}\right)$, and

$$
\begin{aligned}
R(1) & =\left(\varepsilon_{11} \otimes I_{2 n}(0 \oplus e)\right) \wedge \cdots \wedge\left(\varepsilon_{n n} \otimes I_{2 n}(0 \oplus e)\right) \\
& =\left(0 \oplus\left(\varepsilon_{11} \otimes I_{n}\right) e\right) \wedge \cdots \wedge\left(0 \oplus\left(\varepsilon_{n n} \otimes I_{n}\right) e\right) \neq 0 .
\end{aligned}
$$

Thus there is a coefficient $K$ with $P_{K} \neq 0$. It must have only finitely many zeros, and thus we have the desired situation. The same argument applies to $\eta$.

THEOREM 3.7 [14] [9]. Suppose that $V \subseteq W$ are operator spaces, and that $A$ is an injective $C^{*}$-algebra. Then any complete contraction $\varphi: V \rightarrow A$ has a completely contractive extension $\psi: W \rightarrow A$.

Proof. First let us suppose that $A=\mathbf{M}_{n}$. We wish to show that the restriction map

$$
\rho: \mathscr{M}\left(W, \mathbf{M}_{n}\right) \rightarrow \mathscr{M}\left(V, \mathbf{M}_{n}\right)
$$

sends the closed unit ball of $\mathscr{M}\left(W, \mathbf{M}_{n}\right)$ onto that of $\mathscr{M}\left(V, \mathbf{M}_{n}\right)$. It suffices to show that the inclusion map of the preduals

$$
V \otimes_{\mathscr{M}} \mathbf{M}_{n^{*}} \hookrightarrow W \otimes_{\mathscr{M}} \mathbf{M}_{n^{*}}
$$

is isometric. It is clear that this map is norm decreasing. Given $F \in V \otimes_{\mathscr{M}} \mathbf{M}_{n^{*}}$, we denote its norms in the latter and in $W \otimes_{\mathscr{M}} \mathbf{M}_{n^{*}}$ by $\|F\|_{V}$ and $\|F\|_{W}$, respectively, let us suppose that $\|F\|_{W}<1$. From Lemma 3.6 we may choose elements $w \in \mathbf{M}_{n}(W)$ and $g \in \mathbf{M}_{n}\left(\mathbf{M}_{n^{*}}\right)$ such that $\|w\|<1,\|g\|=1$, and $g$ is non-singular with $F=w \times g$. Since $g$ is non-singular, the map

$$
\theta_{g}: \mathbf{M}_{n}(W) \rightarrow W \otimes \mathbf{M}_{n^{*}}: w=\sum w_{i j} \otimes \varepsilon_{i j} \mapsto w \times g=\sum w_{i j} \otimes g_{i j}
$$

is an isomorphism. Thus since $\theta_{g}(w)=F \in V \otimes \mathbf{M}_{n^{*}}=\theta_{g}\left(\mathbf{M}_{n}(V)\right)$, we must have that $w \in \mathbf{M}_{n}(V)$. Noting that $\mathbf{M}_{n}(V) \hookrightarrow \mathbf{M}_{n}(W)$ is assumed isometric, we conclude that $\|F\|_{V} \leq\|w\|\|g\|<1$.

Given an arbitrary injective $C^{*}$-algebra $A \subseteq \mathscr{B}(H)$, there is a complete contraction $\Phi: \mathscr{B}(H) \rightarrow A$. Since we may compose this with a map of $W$ into $\mathscr{B}(H)$, it suffices to consider the case $A=\mathscr{B}(H)$. If $e$ 
is a projection with $\operatorname{dim} e H=n$, then $e \mathscr{B}(H) e \cong \mathbf{M}_{n}$. Thus letting $\mathscr{L}$ be the family of finite dimensional spaces $L \subseteq H$, and letting $e_{L}$ be the projection onto $L$, we may choose for each $L \in \mathscr{L}$ a completely contractive extension $\psi_{L}: W \rightarrow e_{L} \mathscr{B}(H) e_{L}$ of $e_{L} \varphi e_{L}: V \rightarrow e_{L} \mathscr{B}(H) e_{L}$. $\mathscr{L}$ is a directed set under inclusion, hence $\left\{\psi_{L}: L \in \mathscr{F}\right\}$ may be regarded as a net in the unit ball of $\mathscr{M}(W, \mathscr{B}(H))$. Since the latter is compact in the topology of point-weak* convergence, this net has a cluster point $\psi$. It is quickly seen that this is the desired extension of $\varphi$.

4. The Haagerup tensor product and bimodules. Given matricially normed spaces $V$ and $W$, the Haagerup tensor product $V \otimes_{h} W$ is a matricially normed space, which is defined as follows (see [4]). Given $v \in \mathbf{M}_{n, p}(V)$ and $w \in \mathbf{M}_{p, n}(W)$, we define

$$
v \odot w \in \mathbf{M}_{n}(V \otimes W)
$$

by "matrix multiplication":

$$
(v \odot w)_{i j}=\sum_{k} v_{i k} \otimes w_{k j}
$$

The Haagerup norm \|\|$_{h}$ on $\mathbf{M}_{n}(V \otimes W)$ is given by

$$
\|u\|_{h}=\inf \left\{\sum_{\nu}\left\|v^{\nu}\right\|\left\|w^{\nu}\right\|: u=\sum_{\nu} v^{\nu} \odot w^{\nu}\right\},
$$

where the sums are finite, and we choose $v^{\nu} \in \mathbf{M}_{n, p_{\nu}}(V), w^{\nu} \in \mathbf{M}_{p_{\nu}, n}$.

LEMMA 4.1. Given matricially normed spaces $V$ and $W$, the Haagerup norms determine a matricial norm structure on $V \otimes W$.

Proof. Subadditivity is trivial. Let us suppose that $u \in \mathbf{M}_{n}(V \otimes W)$. If

$$
u \oplus 0_{q}=\sum_{\nu} v^{\nu} \odot w^{\nu}, \quad\left(v^{\nu} \in \mathbf{M}_{n+q, p_{\nu}}(V), w^{\nu} \in \mathbf{M}_{p_{\nu}, n+q}(W),\right.
$$

then letting $\left[I_{n} O\right]$,

$$
u=\sum_{\nu} E v^{\nu} \odot w^{\nu} E^{*}
$$

where $\left\|E v^{\nu}\right\| \leq\left\|v^{\nu}\right\|$, and $\left\|w^{\nu} E^{*}\right\| \leq\left\|w^{\nu}\right\|$. It follows that $\|u\|_{h} \leq$ $\|u \oplus 0\|_{h}$. The reverse inequality is trivial. Given $\alpha \in \mathbf{M}_{n}$, and $u=$ $\sum_{\nu} v^{\nu} \odot w^{\nu}$, we have that $\alpha u=\sum_{\nu}\left(\alpha v^{\nu}\right) \odot w^{\nu}$, and thus $\|\alpha u\|_{h} \leq$ $\|\alpha\| \sum_{\nu}\left\|v^{\nu}\right\|\left\|w^{\nu}\right\|$. It follows that $\|\alpha u\|_{h} \leq\|\alpha\|\|u\|_{h}$. Similarly, $\|u \alpha\| \leq$ $\|u\|\|\alpha\|$. 
Finally we have to show that the Haagerup norms indeed have no null space. Given $0 \neq u \in V \otimes W$, let us suppose that $f \in V^{*}$ and $g \in W^{*}$ satisfy $\|f\|=\|g\|=1$. Then given any decomposition $u=$ $\sum_{\nu} v^{\nu} \odot w^{\nu}, v^{\nu} \in \mathbf{M}_{1, p_{\nu}}(V), w^{\nu} \in \mathbf{M}_{p_{\nu}, 1}(W)$, we have that

$$
|f \otimes g(u)| \leq \sum_{\nu}\left|f_{1, p_{\nu}}\left(v^{\nu}\right) \odot g_{p_{\nu}, 1}\left(w^{\nu}\right)\right| \leq \sum_{\nu}\left\|v^{\nu}\right\|\left\|w^{\nu}\right\|,
$$

since we have that $\|f\|_{c b}=\|f\|$ and $\|g\|_{c b}=\|g\|$. Thus $0 \neq\|u\|_{\lambda} \leq$ $\|u\|_{h}$. In general given $0 \neq u \in \mathbf{M}_{n}(V \otimes W)$, let us assume that $u_{i j} \neq 0$. Then letting

we have that

$$
\begin{gathered}
i \\
\downarrow \\
E_{i}=[0 \cdots 1 \cdots 0]
\end{gathered}
$$

$$
0 \neq\left\|u_{i j}\right\|_{h}=\left\|E_{i} u E_{j}^{*}\right\| \leq\|u\|_{h}
$$

In many respects the Haagerup tensor product is analogous to the projective tensor product for normed vector spaces (see $\S 2$ ). In particular, it is natural to define a bilinear map $\varphi: V \times W \rightarrow X$ to be completely bounded if the corresponding linear map $\varphi: V \otimes_{h} W \rightarrow X$ is completely bounded (see [2]). A convincing argument for this point of view was suggested to us by B. E. Johnson. He remarked that the theory of matricially normed spaces might be simplified if one instead considered normed modules over the infinite matrix algebra. We verify below that the corresponding functor transforms the Haagerup tensor product of two matricially normed spaces into the corresponding projective normed bimodule tensor product.

We define $\mathscr{F}$ to be the ${ }^{*}$-algebra of complex matrices with countably many rows and columns, having only finitely many non-zero entries. We may regard $\mathscr{F}$ as the inductive limit of the system of matrix algebras:

$$
\mathbf{M}_{1} \subseteq \mathbf{M}_{2} \subseteq \cdots
$$

where the connecting maps are given by $\alpha \rightarrow \alpha \oplus 0_{1}$. We let $e_{i j}^{n}$ denote the matrix units in $\mathbf{M}_{n}$, and $1_{n}$ the identity for $\mathbf{M}_{n}$. Since the usual operator norms are compatible, we may regard $\mathscr{F}$ as a normed *algebra.

Given a normed algebra $\mathscr{A}$, a normed $\mathscr{A}$-bimodule $V$ is an $\mathscr{A}$ bimodule with a norm satisfying $\|\alpha v\| \leq\|\alpha\|\|v\|$ and $\|v \alpha\| \leq\|v\|\|\alpha\|$ for $v \in V$ and $\alpha \in \mathscr{A}$. Given any matricially normed space $V$, we may 
identify $\mathscr{V}=V \otimes \mathscr{F}$ with the direct limit of the system of normed spaces

$$
V \otimes \mathbf{M}_{1} \subseteq V \otimes \mathbf{M}_{2} \subseteq \cdots .
$$

By condition $M_{1}$, the matricial norms are compatible, and thus determine a norm on $\mathscr{V}=V \otimes \mathscr{F}$. Regarding $\mathscr{V}$ as an $\mathscr{F}$-bimodule, it follows from $M_{2}$ that $\mathscr{V}$ is a normed $\mathscr{F}$-bimodule.

We say that an $\mathscr{F}$-bimodule $\mathscr{V}$ is non-degenerate if for each $v \in \mathscr{V}$, there is an $n \in \mathbf{N}$ such that $1_{n} v=v 1_{n}=v$. Given a non-degenerate $\mathscr{F}$-bimodule $\mathscr{V}$, we let $V=e_{11} \mathscr{V} e_{11}$. We have that $V \neq\{0\}$. To see this suppose that $0 \neq v \in \mathscr{V}$. Letting $n$ be such that $v=1_{n} v 1_{n}$, it follows that there exist $i, j$ with $v^{\prime}=e_{i i} v e_{j j} \neq 0$. Letting $v^{\prime \prime}=e_{1 i} v^{\prime} e_{j 1}$, we have that $v^{\prime}=e_{i 1} v^{\prime \prime} e_{1 j}$, and thus $v^{\prime \prime}$ is a non-zero element of $V$. It is a simple matter to verify that the map

$$
v \mapsto \sum e_{1 i} v e_{j 1} \otimes e_{i j}
$$

determines a bimodule isomorphism $\mathscr{V} \cong V \otimes \mathscr{F}$. If we assume that $\mathscr{V}$ is a non-degenerate normed $\mathscr{F}$-module, then we may let $1_{n} \mathscr{V} 1_{n} \cong$ $V \otimes \mathbf{M}_{n}$ have the relative norm. $V$ is thereby a matricially normed space, and we see that the construction of the previous paragraph essentially gives all of the non-degenerate normed $\mathscr{F}$-bimodules.

If $\varphi: V \rightarrow W$ is a linear map, then the corresponding map $\varphi \otimes$ id: $V \otimes \mathscr{F} \rightarrow W \otimes \mathscr{F}$ is an $\mathscr{F}$-bimodule map, and the $\mathscr{F}$-bimodule maps $\Phi: V \otimes \mathscr{F} \rightarrow W \otimes \mathscr{F}$ are precisely those of the form $\varphi \otimes 1$. This is a consequence of the fact that $\Phi\left(e_{i j} v e_{k l}\right)=e_{i j} \Phi(v) e_{k l}$. Under this construction, the complete contractions $\varphi$ correspond exactly to the contractive $\mathscr{F}$-bimodule maps $\Phi: V \otimes \mathscr{F} \rightarrow W \otimes \mathscr{F}$.

Given non-degenerate normed $\mathscr{F}$-bimodules $\mathscr{V}$ and $\mathscr{W}$ we shall notationally identify the algebraic tensor product $\mathscr{V} \otimes \mathscr{W}=V \otimes \mathscr{F} \otimes$ $W \otimes \mathscr{F}$ with $V \otimes W \otimes \mathscr{F} \otimes \mathscr{F}$. The projective tensor product $\mathscr{V} \hat{\otimes} \mathscr{W}$ (see $\S 2$-we do not complete) is then a normed $\mathscr{F}$-bimodule under the external operations $\alpha(v \otimes w)=\alpha v \otimes w$, and $(v \otimes w) \alpha=v \otimes w \alpha$. Letting $J=J(\mathscr{V} \otimes \mathscr{W})=\left\{\sum_{i} v_{i} \alpha_{i} \otimes w_{i}-v_{i} \otimes \alpha_{i} w_{i}: v_{i} \in \mathscr{V}, w_{i} \in \mathscr{W}, \alpha_{i} \in \mathscr{F}\right\}$, we define the projective $\mathscr{F}$-tensor product by

$$
\mathscr{V} \hat{\otimes} \mathscr{F} \mathscr{W}=(\mathscr{V} \hat{\otimes} \mathscr{W}) / \bar{J},
$$

where the bar indicates the norm closure. Since $\bar{J}$ is a closed (external) $\mathscr{F}$-bimodule, the quotient is a normed non-degenerate $\mathscr{F}$-bimodule. 
Letting $\mathscr{V}=V \otimes \mathscr{F}$ and $\mathscr{W}=W \otimes \mathscr{F}$ be two non-degenerate $\mathscr{F}$ bimodules, we define an external bimodule map

\section{$\Lambda: V \otimes \mathscr{F} \otimes W \otimes \mathscr{F} \rightarrow V \otimes W \otimes \mathscr{F}$}

by letting

$\Lambda(v \otimes \alpha \otimes w \otimes \beta)=v \otimes w \otimes \alpha \beta$.

In general, given $v \in \mathscr{V}, w \in \mathscr{W}$, we let $v \odot w=\Lambda(v \otimes w)$.

THEOREM 4.2. $J(\mathscr{V} \otimes \mathscr{W})=\operatorname{ker} \Lambda$.

Proof. Since we have that

$$
\Lambda(v \otimes \alpha \gamma \otimes w \otimes \beta)=\Lambda(v \otimes \alpha \otimes w \otimes \gamma \beta),
$$

it is evident that $J=J(\mathscr{V} \otimes \mathscr{W}) \subseteq \operatorname{ker} \Lambda$.

Conversely if we are given $\Lambda(u)=0$, where $u=\sum_{i=1}^{p} u_{i} \otimes \alpha_{i} \otimes \beta_{i}$ $\left(u_{i} \in V \otimes W\right)$, it suffices to show that $u \equiv 0(\bmod J)$. We may choose $n \in \mathbf{N}$ with $\alpha_{i}, \beta_{i} \in \mathbf{M}_{n}$ and we define $E_{i j} \in \mathscr{F}(1 \leq k, l \leq p)$ by

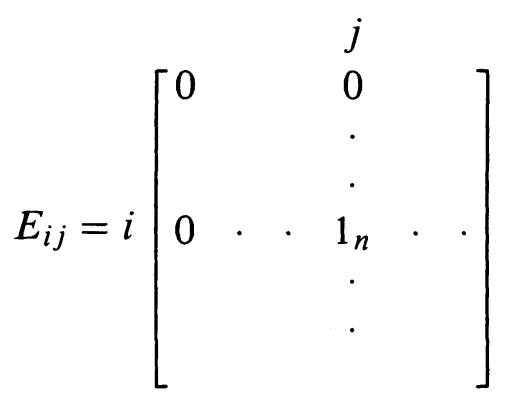

Letting $t=\sum_{i} u_{i} \otimes \alpha_{i} E_{1 i}, \beta=\sum_{j} E_{j 1} \beta_{j}$ we have that

$$
t \beta=\left[\begin{array}{ccc}
u_{1} \otimes \alpha_{1} & u_{2} \otimes \alpha_{2} & \ldots \\
0 & 0 & \ldots
\end{array}\right]\left[\begin{array}{ccc}
\beta_{1} & 0 & \ldots \\
\beta_{2} & 0 & \ldots \\
& \ldots & \ldots
\end{array}\right]=\sum_{i} u_{i} \otimes \alpha_{i} \beta_{i}=0 .
$$

It follows that if $\rho$ is the range projection of $\beta$, i.e., the minimal projection with $\beta=\rho \beta$, then $t \rho=0$. To see this, let $\chi_{(0, \infty)}$ be the characteristic function of the set $(0, \infty)$. Then $\rho=p\left(\beta \beta^{*}\right)$ where

$$
p(X)=\alpha_{1} X+\alpha_{2} X^{2}+\cdots+\alpha_{r} X^{r}
$$

is a real polynomial such that $\left.p\right|_{s p \beta \beta^{*}}=\left.\chi_{(0, \infty)}\right|_{s p \beta \beta^{*}}$. We conclude that

$$
\begin{aligned}
u & =\sum_{i} u_{i} \otimes \alpha_{i} \otimes \beta_{i}=\sum_{i, j} u_{i} \otimes \alpha_{i} E_{1 i} \otimes E_{j 1} \beta_{j} \\
& =t \otimes \beta \equiv t \rho \otimes \beta=0 \quad(\bmod J) .
\end{aligned}
$$


We define a linear map $\theta: V \otimes W \otimes e_{11} \rightarrow \mathscr{V} \otimes \mathscr{W}$ by

$$
\theta\left(v \otimes w \otimes e_{11}\right)=v \otimes w \otimes e_{11} \otimes e_{11} .
$$

This extends uniquely to an $\mathscr{F}$-bimodule map $\theta: V \otimes W \otimes \mathscr{F} \rightarrow \mathscr{V} \otimes \mathscr{W}$ via the formula

$$
\theta\left(v \otimes w \otimes e_{i j}\right)=v \otimes w \otimes e_{i 1} \otimes e_{1 j}
$$

(recall that we let $\mathscr{V} \otimes \mathscr{W}$ have the external module operations). It follows that for all $u \in V \otimes W \otimes \mathscr{F}, \Lambda(\theta(u))=u$. Letting $\pi: \mathscr{V} \otimes W \rightarrow$ $\mathscr{V} \otimes_{\mathscr{F}} \mathscr{W}$ be the quotient map we have that

$$
\pi\left(\theta\left(V \otimes W \otimes e_{11}\right)\right)=e_{11}(\mathscr{V} \otimes \mathscr{F} \mathscr{W}) e_{11}
$$

since if we are given $v \in V, w \in W$,

$$
\begin{aligned}
e_{11}(v \otimes w \otimes \alpha \otimes \beta) e_{11} & =v \otimes w \otimes e_{11} \alpha \otimes \beta e_{11} \\
& =v \otimes w \otimes e_{11} \alpha \beta e_{11} \otimes e_{11} \quad(\bmod \bar{J}),
\end{aligned}
$$

where $e_{11} \alpha \beta e_{11} \in \mathbf{C} e_{11}$. Since $\pi$ is a bimodule map, it follows that $\pi$ maps $\theta(V \otimes W \otimes \mathscr{F})$ surjectively onto $\mathscr{V} \otimes \mathscr{F} \mathscr{W}$. Letting $u \in V \otimes W \otimes \mathscr{F}$, we find that

$$
\begin{aligned}
\| \pi & \theta(u) \|=\inf \left\{\sum\left\|v_{i}\right\|\left\|w_{i}\right\|: \theta(u)-\sum v_{i} \otimes w_{i} \in \bar{J}, v_{i} \in \mathscr{V}, w_{i} \in \mathscr{W}\right\} \\
& =\inf \left\{\sum\left\|v_{i}\right\|\left\|w_{i}\right\|: \theta(u)-\sum v_{i} \otimes w_{i} \in J, v_{i} \in \mathscr{V}, w_{i} \in \mathscr{W}\right\} \\
& =\inf \left\{\sum\left\|v_{i}\right\|\left\|w_{i}\right\|: \Lambda(\theta(u))=\Lambda\left(\sum v_{i} \otimes w_{i}\right), v_{i} \in \mathscr{V}, w_{i} \in \mathscr{W}\right\} \\
& =\inf \left\{\sum\left\|v_{i}\right\|\left\|w_{i}\right\|: u=\sum v_{i} \odot w_{i}, v_{i} \in \mathscr{V}, w_{i} \in \mathscr{W}\right\} .
\end{aligned}
$$

Given operator spaces $V$ and $W$, this coincides with the Haagerup matricial tensor product norms. Thus in that case we have

$$
\left(V \otimes_{h} W\right) \otimes \mathscr{F} \cong \mathscr{V} \hat{\otimes}_{\mathscr{F}} \mathscr{W} .
$$

We conclude this section with a problem. Paulsen and Smith have recently proved that if $V \subseteq V_{1}$ and $W$ are operator spaces, then the inclusion $V \otimes_{h} W \hookrightarrow V_{1} \otimes_{h} W$ is completely isometric [10]. This came as quite a surprise since the projective product for normed vector spaces does not have this property. In fact a Banach space $W$ has the property that $V \hat{\otimes} W \hookrightarrow V_{1} \hat{\otimes} W$ is an isometry for all pairs $V \subseteq V_{1}$ if and only if $W$ is isometric to $L^{1}(X, \mu)$ for some measure space $(X, \mu)$ (see [5]). The inclusion property for $\otimes_{h}$ seems analogous to that given for $\otimes_{\mathscr{M}}$ in $\S 3$. In fact it follows from [4] that if $V$ and $W$ are operator spaces, and $u \in \mathbf{M}_{n n}\left(V \otimes_{h} W\right)$ satisfies $\|u\|<1$, then there exist a 
$p \in \mathbf{N}, v \in \mathbf{M}_{n p}(V), w \in \mathbf{M}_{p n}(W)$ with $\|v\|,\|w\|<1$ and $u=v \odot w$. In this case, however, we do not know if we may take the components of $w$ to be linearly independent. That fact would give an elementary proof of the Paulsen and Smith result. Since it seems unlikely one could maintain equality with the components of $w$ independent, we conjecture that it is rather some perturbation version of this argument that will apply.

5. The completely bounded tensor product and bimodules. Let us suppose that $V$ and $W$ are matricially normed spaces. Extending the discussion of $\S 3$, we define the completely bounded seminorm on $V \otimes W$ by

$$
\|u\|_{\mathscr{M}}=\inf \left\{\sum_{\nu}\left\|v^{\nu}\right\|\left\|w^{\nu}\right\|: u=\sum_{\nu} v^{\nu} \times w^{\nu}\right\},
$$

where the sums are finite, and we choose $v^{\nu} \in \mathbf{M}_{n^{\nu}}(V), w^{\nu} \in \mathbf{M}_{n^{\nu}}(W)$. If $W^{*}$ is an operator space, one may use a generalization of Corollary 3.4 to show that this is a norm, but in general this is not the case. We define the completely bounded tensor product $V \otimes_{\mathscr{M}} W$ to be $(V \otimes W) / N$, where $N$ is the null space of \|\|$_{\mathscr{M}}$.

As in $\S 4$, we let $\mathscr{V}=V \otimes \mathscr{F}$ and $\mathscr{W}=W \otimes \mathscr{F}$. We regard $\mathscr{V}$ (resp. $\mathscr{W}$ ) as a right (resp. left) normed module over the algebraic tensor product $\mathscr{F} \otimes \mathscr{F}$ by letting

$$
v(\alpha \otimes \beta)=\alpha^{\operatorname{tr}} v \beta, \quad(\alpha \otimes \beta) w=\alpha w \beta^{\operatorname{tr}} .
$$

We define the corresponding projective $\mathscr{F} \otimes \mathscr{F}$ tensor product by

$$
\mathscr{V} \hat{\otimes} \mathscr{F} \otimes \mathscr{F} \mathscr{W}=\mathscr{V} \hat{\otimes} \mathscr{W} / \bar{K}
$$

where

$$
\begin{aligned}
K & =K(\mathscr{V} \otimes \mathscr{W}) \\
& =\left\{\sum_{i} v_{i} \gamma_{i} \otimes w_{i}-v_{i} \otimes \gamma_{i} w_{i}: v_{i} \in \mathscr{V}, w_{i} \in \mathscr{W}, \gamma_{i} \in \mathscr{F} \otimes \mathscr{F}\right\},
\end{aligned}
$$

and we denote the quotient map by $\pi$. It should be noted that this tensor product is a normed vector space, but that it does not have a natural bimodule structure.

We have a natural bilinear form $\langle\rangle:, \mathscr{F} \times \mathscr{F} \rightarrow \mathbf{C}$ defined by

$$
\langle\alpha, \beta\rangle=\sum \alpha_{i j} \beta_{i j}
$$

We define a linear map $\Theta: \mathscr{V} \otimes \mathscr{W} \rightarrow V \otimes W$ by letting

$$
\Theta(v \otimes \alpha \otimes w \otimes \beta)=\langle\alpha, \beta\rangle v \otimes w .
$$


Since for all $\alpha, \beta, \gamma, \delta \in \mathscr{F}$

$$
\langle\delta \alpha \varepsilon, \beta\rangle=\left\langle\alpha, \delta^{\operatorname{tr}} \beta \varepsilon^{\mathrm{tr}}\right\rangle,
$$

we have that for all $\gamma \in \mathscr{F} \otimes \mathscr{F}$ and $v \in \mathscr{V}, w \in \mathscr{W}$

$$
\Theta(v \gamma \otimes w)=\Theta(v \otimes \gamma w) \text {. }
$$

Defining $v \times w=\sum v_{i j} \otimes w_{i j}$ for $v \in \mathscr{V}$ and $w \in \mathscr{W}$, we have $\Theta(v \otimes w)=$ $v \times w$.

THEOREM 5.1. $K(\mathscr{V} \otimes \mathscr{W})=\operatorname{ker} \Theta$.

Proof. From (5.1), the inclusion $K(\mathscr{V} \otimes \mathscr{W}) \subseteq \operatorname{ker} \Theta$ is immediate. Conversely, let us suppose that $u=\sum_{k} v_{k} \otimes \alpha^{k} \otimes w_{k} \otimes \beta^{k} \in \operatorname{ker} \Theta$, where $v_{k} \in V, w_{k} \in W$. Then

$$
0=\Theta(u)=\sum_{k}\left\langle\alpha^{k}, \beta^{k}\right\rangle v_{k} \otimes w_{k}
$$

Since $\varepsilon_{i j}=\varepsilon_{i 1} \varepsilon_{11} \varepsilon_{1 j}$, we have modulo $K$,

$$
\begin{aligned}
u & =\sum_{i, j, k} \alpha_{i j}^{k} v_{k} \otimes \varepsilon_{i j} \otimes w_{k} \otimes \beta^{k} \\
& =\sum_{i, j, k} \alpha_{i j}^{k}\left[\left(v_{k} \otimes \varepsilon_{11}\right)\left(\varepsilon_{1 i} \otimes \varepsilon_{1 j}\right)\right] \otimes\left[w_{k} \otimes \beta^{k}\right] \\
& =\sum_{i, j, k} \alpha_{i j}^{k}\left[\left(v_{k} \otimes \varepsilon_{11}\right)\right] \otimes\left[\left(\varepsilon_{1 i} \otimes \varepsilon_{1 j}\right)\left(w_{k} \otimes \beta^{k}\right)\right] \\
& =\sum_{i, j, k} \alpha_{i j}^{k}\left[\left(v_{k} \otimes \varepsilon_{11}\right)\right] \otimes\left[\left(w_{k} \otimes \beta_{i j}^{k} \varepsilon_{11}\right)\right] \\
& =\sum_{k}\left\langle\alpha^{k}, \beta^{k}\right\rangle v_{k} \otimes \varepsilon_{11} \otimes w_{k} \otimes \varepsilon_{11}=0,
\end{aligned}
$$

since the map $V \otimes W \rightarrow \mathscr{V} \otimes \mathscr{W}: v \otimes w \mapsto v \otimes \varepsilon_{11} \otimes w \otimes \varepsilon_{11}$ is linear. Thus $u \in K(\mathscr{V} \otimes \mathscr{W})$.

We define $\psi: V \otimes W \rightarrow \mathscr{V} \hat{\otimes} \mathscr{W}$ by $\psi(v \otimes w)=v \otimes \varepsilon_{11} \otimes w \otimes \varepsilon_{11}$. It is

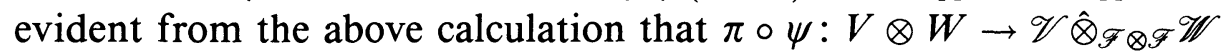
is surjective. The quotient norm is given by

$$
\begin{aligned}
\|\pi \psi(u)\|=\inf \left\{\sum\left\|v_{i}\right\|\left\|w_{i}\right\|: \psi(u)-\sum v_{i} \otimes w_{i} \in \bar{K}, v_{i} \in \mathscr{V}, w_{i} \in \mathscr{W}\right\} \\
\quad=\inf \left\{\sum\left\|v_{i}\right\|\left\|w_{i}\right\|: \psi(u)-\sum v_{i} \otimes w_{i} \in K, v_{i} \in \mathscr{V}, w_{i} \in \mathscr{W}\right\} \\
\quad=\inf \left\{\sum\left\|v_{i}\right\|\left\|w_{i}\right\|: \Theta \psi(u)=\Theta\left(\sum v_{i} \otimes w_{i}\right), v_{i} \in \mathscr{V}, w_{i} \in \mathscr{W}\right\} \\
\quad=\inf \left\{\sum\left\|v_{i}\right\|\left\|w_{i}\right\|: u=\sum v_{i} \times w_{i}, v_{i} \in \mathscr{V}, w_{i} \in \mathscr{W}\right\} \\
=\|u\|_{\mathscr{M}}
\end{aligned}
$$


We conclude that we have a natural isometry:

$$
V \otimes_{\mathscr{M}} W=\mathscr{V} \hat{\otimes}_{\mathscr{F} \otimes \mathscr{F}} \mathscr{W} .
$$

Added in proof. It follows from [15, Theorem 3.3], that any complete $L^{1}$-matricially normed space is a quotient of $\mathscr{B}(H)_{*}$ for some Hilbert space $H$. This generalization of Corollary 2.3 may be used to simplify the proof of Theorem 2.4.

\section{REFERENCES}

[1] W. Arveson, Subalgebras of $C^{*}$-algebras, Acta Math., 123 (1969), 141-224.

[2] E. Christensen and A. Sinclair, Representations of completely bounded k-linear operators, J. Funct. Analysis, 72 (1987), 151-181.

[3] J. Dixmier, Les Algèbres d'Opérateurs dans l'Espace Hilbertien, 2nd ed., Cahiers Scientifiques vol 25, Gauthier-Villars, Paris, 1969.

[4] E. Effros and A. Kishimoto, Module maps and $C^{*}$-algebraic cohomology, Indiana Univ. Math. J., 36 (1987), 257-276.

[5] A. Grothendieck, Une characterisation vectorielle-metrique des espaces $L^{1}$, Canad. J. Math., 7 (1955), 552-561

[6] U. Haagerup, Decomposition of completely bounded maps, unpub. ms.

[7] R. Kadison, Isometries of operator algebras, Ann. of Math., 54 (1951), 325-338.

[8] E. Lacey, The Isometric Theory of Classical Banach Spaces, Grundlehren die Math. Wiss. vol. 28, Springer-Verlag, NY 1974.

[9] V. Paulsen, Completely bounded maps on $C^{*}$-algebras and invariant operator ranges, Proc. Amer. Math. Soc., 86 (1982), 91-96.

[10] V. Paulsen and R. Smith, Multilinear maps and tensor norms on operator systems, J. Funct. Analysis, 73 (1987), 258-276.

[11] Z.-J. Ruan, Subspaces of $C^{*}$-algebras, to appear.

[12] R. Smith, Completely bounded maps between $C^{*}$-algebras, J. London Math. Soc., (2) 27 (1983), 157-166.

[13] M. Takesaki, Theory of Operator Algebras, I, Springer-Verlag, New York.

[14] G. Wittstock, Ein operatorwertiger Hahn-Banach Satz, J. Funct. Analysis, 40 (1981), 127-150.

[15] E. Effros and Z. J. Ruan, Representations of operator bimodules and their applications, to appear.

Received February 15, 1987. The first author was supported in part by NSF. The second author was supported in part by the Sloan Foundation.

UNIVERSITY OF CALIFORNIA

LOS ANGELES, CA 90024-1555 


\title{
PACIFIC JOURNAL OF MATHEMATICS EDITORS
}

\author{
V. S. VARADARAJAN \\ (Managing Editor) \\ University of California \\ Los Angeles, CA 90024 \\ HERBERT Clemens \\ University of Utah \\ Salt Lake City, UT 84112 \\ R. FINN \\ Stanford University \\ Stanford, CA 94305
}

\author{
HERMANN FLASCHKA \\ University of Arizona \\ Tucson, AZ 85721
}

Ramesh A. Gangolli University of Washington Seattle, WA 98195

VAUGHAN F. R. JONES University of California Berkeley, CA 94720
ROBION KIRBY

University of California

Berkeley, CA 94720

C. C. MOORE

University of California

Berkeley, CA 94720

HAROLD STARK

University of California, San Diego La Jolla, CA 92093

\section{ASSOCIATE EDITORS}

\author{
R. ARENS \\ E. F. BECKENBACH \\ B. H. NEUMANN \\ F. WOLF \\ K. YOSHIDA \\ (1906-1982)

\section{SUPPORTING INSTITUTIONS}

\section{UNIVERSITY OF ARIZONA} \\ UNIVERSITY OF BRITISH COLUMBIA \\ CALIFORNIA INSTITUTE OF TECHNOLOGY \\ UNIVERSITY OF CALIFORNIA \\ MONTANA STATE UNIVERSITY \\ UNIVERSITY OF NEVADA, RENO \\ NEW MEXICO STATE UNIVERSITY \\ OREGON STATE UNIVERSITY \\ UNIVERSITY OF OREGON \\ UNIVERSITY OF SOUTHERN CALIFORNIA \\ STANFORD UNIVERSITY \\ UNIVERSITY OF HAWAII \\ UNIVERSITY OF TOKYO \\ UNIVERSITY OF UTAH \\ WASHINGTON STATE UNIVERSITY \\ UNIVERSITY OF WASHINGTON
}

The Supporting Institutions listed above contribute to the cost of publication of this Journal, but they are not owners or publishers and have no responsibility for its content or policies.

Mathematical papers intended for publication in the Pacific Journal of Mathematics should be in typed form or offset-reproduced (not dittoed), double spaced with large margins. Please do not use built up fractions in the text of the manuscript. However, you may use them in the displayed equations. Underline Greek letters in red, German in green, and script in blue. The first paragraph must be capable of being used separately as a synopsis of the entire paper. In particular it should contain no bibliographic references. Please propose a heading for the odd numbered pages of less than 35 characters. Manuscripts, in triplicate, may be sent to any one of the editors. Please classify according to the scheme of Math. Reviews, Index to Vol. 39. Supply name and address of author to whom proofs should be sent. All other communications should be addressed to the managing editor, or Elaine Barth, University of California, Los Angeles, California 90024

There are page-charges associated with articles appearing in the Pacific Journal of Mathematics. These charges are expected to be paid by the author's University, Government Agency or Company. If the author or authors do not have access to such Institutional support these charges are waived. Single authors will receive 50 free reprints; joint authors will receive a total of 100 free reprints. Additional copies may be obtained at cost in multiples of 50 .

The Pacific Journal of Mathematics is issued monthly as of January 1966. Regular subscription rate: $\$ 190.00$ a year (5 Vols., 10 issues). Special rate: $\$ 95.00$ a year to individual members of supporting institutions.

Subscriptions, orders for numbers issued in the last three calendar years, and changes of address should be sent to Pacific Journal of Mathematics, P.O. Box 969, Carmel Valley, CA 93924, U.S.A. Old back numbers obtainable from Kraus Periodicals Co., Route 100, Millwood, NY 10546.

The Pacific Journal of Mathematics at P.O. Box 969, Carmel Valley, CA 93924 (ISSN 0030-8730) publishes 5 volumes per year. Application to mail at Second-class postage rates is pending at Carmel Valley, California, and additional mailing offices. Postmaster: send address changes to Pacific Journal of Mathematics, P.O. Box 969, Carmel Valley, CA 93924.

PUBLISHED BY PACIFIC JOURNAL OF MATHEMATICS, A NON-PROFIT CORPORATION Copyright (C) 1988 by Pacific Journal of Mathematics 


\section{Pacific Journal of Mathematics}

\section{Vol. 132, No. $2 \quad$ February, 1988}

Jeffery Marc Bergen and Luisa Carini, A note on derivations with power central values on a Lie ideal ..............................209

Alfonso Castro and Sumalee Unsurangsie, A semilinear wave equation

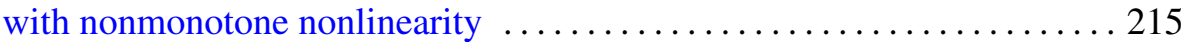

Marius Dadarlat, On homomorphisms of matrix algebras of continuous

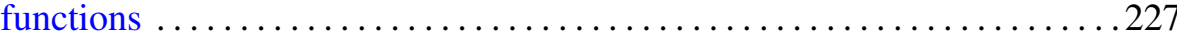

A. Didierjean, Quelques classes de cobordisme non orienté refusant de se

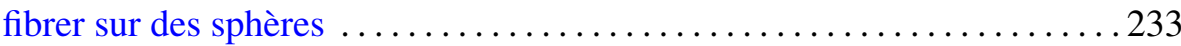

Edward George Effros and Zhong-Jin Ruan, On matricially normed spaces

Sherif El-Helaly and Taqdir Husain, Orthogonal bases are Schauder bases

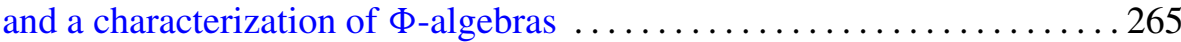

Edward Richard Fadell and Peter N-S Wong, On deforming $G$-maps to be

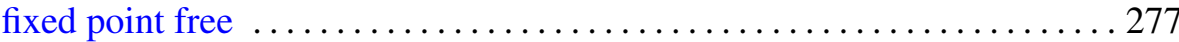

Jean-Jacques Gervais, Stability of unfoldings in the context of equivariant

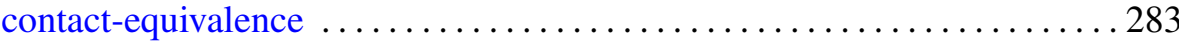

Douglas Martin Grenier, Fundamental domains for the general linear group

Ronald Scott Irving and Brad Shelton, Loewy series and simple projective

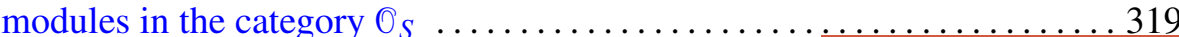

Russell Allan Johnson, On the Sato-Segal-Wilson solutions of the K-dV equation

Thomas Alan Keagy and William F. Ford, Acceleration by subsequence transformations

Min Ho Lee, Mixed cusp forms and holomorphic forms on elliptic varieties

Charles Livingston, Indecomposable surfaces in 4-space 